Gegenüber der Thatsache, dass Glycogen bei sehr langer Behandlung mit verdünnter Kalilösung in der Kälte oder unter kuirzer dauernder Behandlung bei höherer Temperatur nicht weniger als $1 / 5$ seines urspringlichen Gewichts verlieren kann, bei welchem Vorgange zugleich die wohl constatirten Veränderungen, nämlich der Verlust der Opalescenz und die Aenderung seines Drehungsvermögens sich geltend machen: entsteht nun die Frage, was mit dem $1 / 5$ des ursprünglichen Glycogens geschehen sei. An eine einfache Wasserentziehung ist kaum zu denken; eher wäre die Vermuthung zulässig, dass ein oder mehrere Körper sich abspalten, welche im Alkohol-Filtrat enthalten sein müssten. Wir wollten auf diese Frage weiter nicht eingehen, da es uns genïgend war nachgewiesen zu haben, dass verdünnte Kalilösungen auf das Glycogen eine nicht unbedeutende Wirkung zu äussern vermögen.

(Aus dem Laboratorium der Klinik des Herrn Geheimrath Leyden.)

\title{
Beitrag zur Lehre von der Glycogenbildung in der Leber.
}

Von

Dr. Jaques Mayer,

practischem Arzt in Karlsbad.

Die physiologisehen Bedingungen für die Bildung und Aufspeicherung des Glycogens in der Leber sind bereits von zahlreichen Forschern geprifft worden, und hat die Literatur in dieser Beziehung namentlich in den; letzten Jahren eine erhebliche Bereicherung erfahren.

Nachdem Claude Bernard und Hensen unabhängig von einander dasselbe in der Leber entdeckt hatten, wurden Fütterungs- 
J. Mayer: Beitrag zur Lehre von der Glycogenbildung von der Leber. 165

versuche der mannigfachsten Art an Thieren vorgenommen, die den Zweck hatten, die Bedingungen für die Entstehung dieser Substanz kennen zu lernen. Nebst Bernard waren es Schmidt, Pavy, Tscherin off u. m. A., die sich dieser Aufgabe unterzogen, und in neuester Zeit, seitdem man durch die Brücke'sche Methode in den Stand gesetzt worden war, das Glycogen mit möglichst geringen Fehlerquellen darzustellen und quantitativ zu bestimmen, hat sich die Zahl dieser Untersuchungen wesentlich vermehrt. Sehr werthvolle Arbeiten auf diesem Gebiete lieferten Luchsinger, G. Salomon, Dock, Weiss, Kïlz, v. Mering, Naunyn, Böhm und Hoffmann, Schöpffer u. m. A. Man ist hierdurch in der That einen erheblichen Schritt weiter gelangt in der Kenntniss von dem Glycogen bildenden Werth nicht nur der Kohlehydrate, sondern auch vieler anderer, physiologisch wichtiger Substanzen.

Weniger klar aber sind unsere Anschaungen uber die Art der Bildung dieser Substanz in den Leberzellen selbst. Cl. Bernard ${ }^{1}$ ) vindicirt der Leber bekanntlich zwei einander entgegengesetzte Funktionen: Eine chemische, d. i. die Umwandlung des Glyeogens in Zucker, und eine vitale, die in der Produktion, Secretion des Glycogens besteht.

Seiner Ansicht nach übt das Nervensystem einen grossen Einfluss auf diese beiden Prozesse. Ersterer, bebauptet er, wäre als Phänomen der Zerstörung anzusehen und stehe unter dem Einflusse des cerebrospinalen Centrums, letzterer, ein Phänomen der Synthese, unter dem Einflusse des Sympathicus. Seiner Ansicht nach coincidirt in jedem Organ des Körpers Funktionssteigerung mit beschleunigter, und Funktionsschwäche mit verlangsamter Circulation.

Für diese beiden Circulationsarten nimmt er auch die beiden erwähnten Nervensysteme in Anspruch. Die Vasomotoren des Sympathicus verlangsamen die Circulation, die des cerebrospinalen Apparates beschleunigen dieselbe. Ein Beispiel hierfür wäre die Submaxillardrïse. Die normale Circulation wird durch Fäden des Sympathicus beherrscht, die beschleunigte von einem Nerven des cerebrospin. Centrums, der Chorda tympani.

1) Leçons sur le diabète. 1877. p. 307 u. 308. 
Dieselben Verhältnisse, nimmt er an, finden sich in der Leber vor: Vasoconstrictoren, welche rom Sympathicus, and Vasodilatatoren, die vom cerebrospinalen Centrum kommen.

Das Analogon der Chord. tymp., das die Circulation und die zuckerbildende Funktion der Leber beschleunigt, habe seinen Ausgangspunkt im Boden des vierten Ventrikels, gehe von da durch das Mark bis zum Niveau des ersten Riickenwirbels, trete daselbst aus, um direct in die Leber zu gelangen.

Dieser Nerv ist es, durch dessen Reizung beschleunigte Lebercirculation und künstlicher, voribergehender Diabetes erzeugt wird.

Wenn er hingegen das Rïckenmark zwischen letztem Halsund erstem Brustwirbel verletzte, fand er das Blut arm an Zucker und die Leber reich an Glycogen. Andererseits ist es Schiff gelungen, durch Durchschneidung des Rückenmarkes in verschiedenen Höhen Diabetes zu erzeugen, was nach der Bernard'schen Theorie offenbar die Verminderung des Glycogens zur Folge haben müsste.

Aus diesen Thatsachen entnehmen wir, dass ausser der Nahrung auch andere Factoren im Organismus die Glycogenbildung beeinflussen mïssen. Zudem haben die neuesten Arbeiten von Pflüger ${ }^{1}$ ), Golz ${ }^{2}$, Heidenha in ${ }^{3}$ ) and Luchsinger ${ }^{4}$ ) für die Beurtheilung der Circulations- und Secretions-Verhältnisse im Organismus uns neue, den B ernard'schen entgegenstehende Gesichtspunkte erschlossen.

Während man früher geglaubt hat, dass die Reflexvorgänge an den Gefässen nur durch die medulla oblongata bewerkstelligt werden, ist man jetzt der Ansicht, dass reflectorische Verengerung erzielt werden kann, selbst wenn das ganze Gehirn sammt medull. oblong. abgetrennt ist. P flüger hat die Nervenendigungen der Leber durchforseht und gefunden, dass der Nerv die Membran der

1) Ueber die Abhängigkeit der Leber von dem Nervensystem. Dieses Arch. Bd. II. p. 459.

2) Ueber gefässerweiternde Nerven. D. Archiv. Bd. IX. u. Bd. XI.

3) D. Arch. Bd. V.

4) Zur Innervation der Gefässe. D. Arch. Bd. XIII. S. 197. Zur Theorie der Secretionen. D. Arch. Bd. XIII. S. 212. 
Leberzelle durchbohrt, und die Axencylinder sich in dem Protoplasma verlieren, und hält es für höchst wahrscheinlich, dass die Nervenfasern die Vorgänge in den Drüsenzellen unmittelbar und nicht durch $Z$ wischenglieder, etwa durch Aenderungen in der Blutcirculation beeinflussen. (d. Archiv. Bd. II. Ueber die Abhängigkeit der Leber von dem Nervensystem p. 459) und Golz, dem es gelungen ist, $\mathrm{zu}$ beweisen, dass das Ritckenmark in seiner ganzen Länge selbstständige Centren für den Gefässtonus enthält (Ueber die Functionen des Lendenmarkes des Hundes, d. Arch. Bd. VIII p. 485), stellt als Resultat seiner Untersuchungen „Ueber gefäss-erweiternde Nerven" als unzweifelhaft fest, dass der tonus der Gefässe der Hauptsache nach von gewissen selbstständigen Endvorrichtungen abhängt, die in den Gefässen selbst oder in deren unmittelbaren Nähe liegen.

Angeregt durch diese interessanten Thatsachen hatte ich mir für dieses Wintersemester die Aufgabe gestellt, den Einfluss des Rückenmarks auf die Glycogenbildung zu studiren.

Es lag in meinem Plane, das Riuckenmark hungernder Kaninchen vom 5. Halswirbel ab seine ganze Länge hindurch in verschiedenen Höhen zu durchschneiden und nach darauf folgender Traubenzucker-Injection in die Vena jugularis den Effect auf die Glycogen-bildende Function der Leber zu prüfen. Es hat sich jedoch bald herausgestellt, dass noch andere Voruntersuchungen nöthig waren. Ich hielt es nämlich für zweckmässig, vorerst eine Reihe von quantitativen Blutbestimmungen sowohl an gefütterten, als hungernden Kaninchen za machen, um mir ein klares Urtheil über die diesen Gegenstand betreffende Frage zu verschaffen, ferner musste dem pathologischen Experimente der Rückenmarksdurchschneidung eine Versuchsreihe von physiologischen Experimenten vorhergehen.

Zwar waren die Resultate, die Schöpffer in seiner Arbeit (Beiträge zur Kenntniss der Glycogenbildung in der Leber, Archiv für experiment. Pathol. Bd. 1) mittheilt, von der Art, dass Traubenzucker-Injectionen in eine Körpervene kein nennenswerthes Ergebniss für meine Untersuchungen erwarten liessen. Schöpffer operirte an ätherisirten Kaninchen, die vorher nicht gehungert hatten, in der Weise, dass er zu je zwei comparativen Versuchen ein Versuchsthier bentitzte. An einem Tage injicirte er in die 
Vena cruralis, am andern Tage in einen Zweig der vena mesenterica gleiche Quantitäten einer 15 prozentigen Traubenzuckerlösung. Im ersteren Falle wurde fast sämmtlicher Zucker im Harn wieder ausgeschieden, während im letzteren Falle keiner, oder nur Spuren davon wieder erschienen. Begreiflich ist es, dass der in die Pfortader injicirte Zucker in der Leber zuriickgehalten wurde, da die Flïssigkeitsmenge mässig war, und die Injectionen langsam bewerkstelligt wurden; auffällig erschien es jedoch bei der bekannten Thatsache, dass zwischen Lebervenen- und Pfortaderblut eine beständige oscillatorische Bewegtng stattfindet, dass der in die Cruralis injicirte Traubenzucker fast vollständig im Harn wieder erschien. Cl. Bernard ${ }^{1}$ ) hat allerdings bei der Injection einer Rohrzuckerlösung in eine Körpervene dieselbe Erfahrung gemacht, aber zu einem ganz andern Resultate gelangte er, wenn er Traubenzueker injicirte. Wenn man, sagt er, in die Vena jugularis eines Hundes eine gewisse Quantität Traubenzucker einspritzt, so erscheint dieser leichter im Harn wieder, als wenn man die gleiche Quantität in einen Zweig der Vena port. injicirt. In dem „leichter erscheinen" ist eben einer Fülle von Möglichkeiten Raum gegeben, und diese Möglichkeiten im Zusammenhang mit dem Schicksale des im Körper zurückgebliebenen Zuckers zu prüfen, wird unter Anderm anch die Aufgabe dieser Arbeit sein.

\section{Zur Methode}

I. des physiologischen Experimentes

(des Experimentes an vorher nicht operirten Thieren).

Es wurden hierzu Kaninchen verwendet, die ohne Ausnahme kräftig und schwer waren; die geringste Hungerzeit war 4, oft aber auch 5 Tage, während welcher ihnen täglich kleine Quantitäten Wassers verabreicht wurden. Nach der erwähnten 4-5 tägigen Hungerzeit wurde der Harn durch Abpressen entleert, das Thier gewogen, aufs Kaninchenbrett gebunden und in die blosgelegte Vene vermittelst einer graduirten Kittel'schen Spritze von genau 20 grm Inhalt Injectionen von einer $10 \%$ Traubenzuckerlösung gemacht. Um die ruhige Handhabung zu sichern, warde die Spritze

1) Leçons sur le diabète. p. 314 und 320 . 
durch die Schraubenklammer eines festen Stativ's, welche erstere nach allen Richtungen beweglich war, festgehalten. Diese Vorrichtung setzte mich in den Stand, der Gefahr einer Zerreissung der Gefässwandung durch die Canüle bei einer plötzlichen Bewegung des Thieres zu begegnen, da von der Beruhigung des letzteren durch Narkose Abstand genommen wurde, um den Zuckergehalt des Blutes nicht zu modifieiren. Regelmässig wurden $40 \mathrm{grm}$. der Lösung eingespritzt, ausnahmsweise auch 38 und $39 \mathrm{grm}$.

Auf die Einspritzung wurden 18-22 Minuten verwendet. Die Flüssigkeit war vorher nicht erwärmt worden. Um das Thier vor Wärmeverlust zu schüitzen, wurde dasselbe in ein warmes Luftmedium von $28-30^{\circ}$ gebracht; hierza diente ein Blechkasten mit doppelten Boden- und Seitenwandungen, in deren Zwischenraum sich Wasser befand, welch letzteres durch eine Gasflamme auf eine beliebige Temperatur gebracht werden konnte. Die Lufttemperatur wurde durch ein am Deckel angebrachtes Thermometer ermittelt, während die Eigenwärme des Thieres durch ein in den Mastdarm eingeführtes Thermometer controlirt werden konnte. Sowohl an dem vordern Ende des Deckels als auch an der demselben entsprechenden Vorderwand waren Ausschnitte angebracht, aus denen Kopf and Hals des Thieres hervorragen konnten. Nachdem letzteres 3-4 Stunden in nicht gefesseltem Zustande gelegen hatte, wurde dasselbe herausgenommen (es hatte in der Regel die normale Mastdarm-Temperatur von 39--39,30). Der Harn wurde durch Druck entleert und aufbewahrt, aus der Carotis, beziehungsweise Jugularis kleine Quantitäten Blutes abgelassen, in einer mit der entsprechenden Quantität schwefelsauren Natrons versehenen, tarirten Porcellanschale bei Seite gestellt, und das Thier durch Halsschnitt getödtet. Das Abdomen wurde nun durch einen Schnitt in der linea alba eröffnet, die Leber rasch entfernt und in bereit stehendes kochendes Wasser gebracht, um nach der Brü cke'schen Methode bestimmt $\mathrm{zu}$ werden. Ueber die Untersuchungsmethoden a) des Harns, b) des Glycogens, c) des Blutes werde ich an anderer Stelle berichten. In der folgenden Tabelle mögen die Resultate einer Versuchsreihe in allen ihren Details ersichtlich gemacht werden. 


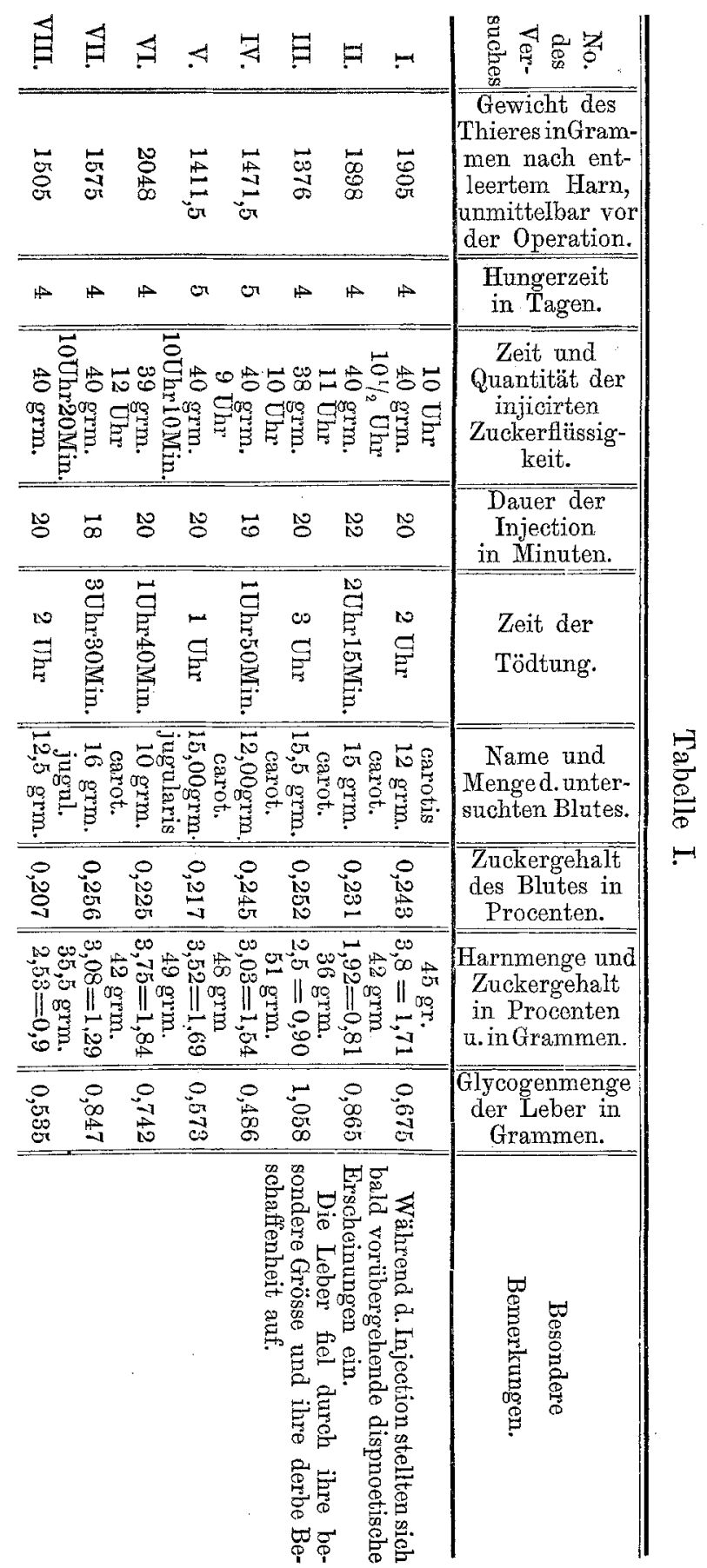


Bei näherer Prüfung dieser Tabelle finden wir, dass von dem in den Körperkreislauf gelangten Zucker, der für die meisten Fälle 4 grm. betrug; durchschnittlich nur der dritte Theil im Harn zur Ausscheidung gelangte, während der iibrige Theil im Organismus zurïckgehalten wurde. Ueberraschend gross zeigen sich die Zahlen für das Glycogen, dessen Umrechnung in Zucker (100 grm. Glycogen $=111,11$ Zucker) einfach ist. Was den Zuckergehalt des Blites anbelangt, kann die Bereehnung desselben gleichfalls nicht schwer fallen, wenn wir die von Dr. Steinberg gefundenen Zahlen für das Verhältniss der Blutmenge zum Körpergewicht bei Kaninchen acceptiren: $1: 12,3-13,3$; ich werde im weitern Verlaufe dieser Arbeit mich des Schlüssels 1:13 bedienen. Bemerken muss ich jedoch schon jetzt, dass der im Blute nachgewiesene Zucker keinesweges auf Rechnung der injicirten Zuckermenge allein gesetzt werden kann.

Ohne zu weit gehende Schlïsse aus den Resultaten dieser Versuchsreihe ziehen zu wollen, lassen sich als unzweifelhaft zwei Thatsachen feststellen:

1) Glycogenfreie Leberzellen brauchen nicht von einem in Folge directer Zufuhr durch. die Pfortader gleichsam mit Zucker überladenen Blute umgeben zu sein, um Glycogen zu bilden, sondern es genügen kleinere Zuckerquantitäten dazu, die von dem auf den ganzen Körperkreislauf vertheilten Zucker dem Leberblute zufallen.

2) Da nach den Untersuchungen von Ludwig und Scheremetjews ky ${ }^{1}$ ) als erwiesen angenommen werden kann, dass der in den Blutkreislauf eingeführte Zucker durch den Sauerstoff des alkalischen Blutes nicht verbrannt wird, so ergiebt sich ferner aus meinen Versuchen, dass ausser der Leber und dem Blute auch andere Orte im Organismus des Thieres vorhanden sein müssen, wo der Zucker als solcher oder in Form von Glycogen aufgespeichert wird.

In der Lymphe hat v. Mehring ${ }^{2}$ ) erst kürzlich den constan-

1) Berichte der Königlichen Gesellsch. d. Wissenschaften. Math. phy. Cl. 1869. Bd. XX. S. 154 und Arbeiten aus der physiolog. Anstalt zu Leipzig. 3. Jahrg. 1868. S. 114.

2) Arch. für Anatom. und Physiolog. 1877. „Ueber die Abzugswege des Zuckers aus der Darmhöhle" von v. Mehring. 
ten Zuckergehalt nachgewiesen, und was das Glycogen anbelangt, so wissen wir ja, dass es ein normaler Bestandtheil der Muskulatur ist. Böhm und $\mathrm{Ho}$ ffmann haben in ihrer Arbeit ${ }^{1}$ ) („Beiträge zur Kenntniss des Kohlehydrat-Stoffwechsels") für den Muskelglycogen-Gehalt von Katern mit einem Körpergewicht von 2-4 Kilo nach Fleischfutterung erhebliche Werthe gefunden, in einem Falle sogar bei 4,07 Kil. Körpergewicht 6,7 grm. Glycogen. $\mathrm{K}$ iihne ${ }^{2}$ ) hat es in den Hoden von Mensehen und Hunden und in den pneumonischen Lungen nachgewiesen. $\mathrm{Jaffe}^{3}$ ) fand eine dem Glycogen ähnliche Substanz im menschlichen Gehirn, während G. Salomon ${ }^{4}$ ) es zweimal im Eiter chronischer Abscesse, im Blute, insbesondere im leukämischen und Naunyn ${ }^{5}$ ) in dem Pfortaderblute mit Amylaceen gefutterter Thiere eine Substanz gefunden hat, welche mit dem Speichelfermente sich in Zucker umwandelte.

Ob das Glyeogen der Musculatur aus der Leber dahin gelangt, oder aber, ob dasselbe dort nnmittelbar aufgespeichert wird, ist eine schwer zu entscheidende Frage, doch möchte ich mich für meinen Theil zu letzterer Ansicht bekennen, da ich mir nicht gut denken kann, dass das so schwer diffundirende Glycogen (nach Bernard ${ }^{6}$ ) und $S c h$ iff $^{7}$ ) ist das Glycogen in Form von eckigen blassen Körnchen in der Leberzelle abgelagert, während Bock und Hoffmann ${ }^{8}$ ) ihm einen amorphen Charakter zuschreiben) in den Blutstrom gelangen und durch diesen ganz unversehrt den Muskeln und andern Organen zugefuhrt werden sollte.

Bevor ich zur Erörterung der weiteren Experimente übergehe, sei es mir gestattet, über die Untersuchungsmethoden des Harns, Glycogens und Blutes einige Bemerkungen zu machen.

1) Arch. für experiment. Patholog. und Pharmak. Bd. VIII.

2) Arch. f. patholog. Anatomie. Bd. XXXII. S. 536.

3) ebenda Bd. XXXVI.

4) Börner's Deutsche Med. Wochenschrift No. 8. 1877 u. No. 35 desselb. Jahrgangs.

5) Beitrag zur Lehre v. Diab. mell. Arch. f. expr. Patholg. P. 92.

6) Compt. rend. 1859. T. XLVIII. p. 884.

7) Schiff, Untersuchungen über die Zuckerbildung in der Leber. Würzburg 1859. - Archiv f. physiol. Heilkunde. N. F. Bd. I. p. 263.

8) Arch. f. path. Anatomie. 1872. Bd. LVI. p. 201. 
a. Harn. Derselbe wurde Anfangs mit Fehlingslösung und vergleichshalber mit dem Wilde'schen Polaristrobometer, später jedoch ausschliesslich nach ersterer Methode untersucht. bestimmt.

b. Glycogen. Dieses wurde nach der Brücke'schen Methode

Die Leber, aus dem abdomen entfernt, wurde in kochendes Wasser gebracht, in demselben mit einer Scheere zerstückelt, die Leberstiicke mit Sand zerrieben, 8-10 Minuten (diese Zeit ist nach meinen Erfahrungen vollständig gentigend) in dem vorher abgegossenen Wasser gekocht, auf das grobe Leinwandfilter gebracht, und der Leberbrei so lange mit kochendem Wasser ausgezogen, bis keine Spur von Opalescenz mehr sichtbar war. Das gesammte Filtrat wurde nun auf $150-250 \mathrm{~cm}$ eingedampft und abwechselnd mit Quecksilberjodkalinm und Salzsäure gefällt, nach einigen Minuten filtrirt, und das Filtrat mit seinem dreifachen Volumen Alkohol versetzt. Nach längerer oder kürzerer Zeit wurde nun decantirt, das Glycogen auf ein Filter gebracht, nochmals mit gewöhnlichem und dann mit absolutem Alkohol mehrere Male ausgewaschen und schliesslich mit Aether extrahirt und bei 110-115 Graden getrocknet. Das Glycogen wurde zwar nicht jedesmal, doch sehr oft auf seine Reactionen geprïft.

Was die Zuckerbestimmung des Blutes anbelangt, so möchte ich an dieser Stelle einige Untersuchungen, die ich in dieser Beziehung angestellt habe, kurz erwähnen.

Die nunmehr als allgemein anerkannte Thatsache, dass das Blut auch unter physiologischen Verhältnissen zuckerhaltig ist, konnte ich auf Grundlage der Bestimmungen, die ich an CarotisBlut von Kaninchen nach der bekannten Methode von Cl. Bernard gemacht habe, bestätigen. Die Resultate stimmen mit denen von Bock und Hoffmann ${ }^{1}$, die gleichfalls das Carotis-Blut von Kaninchen untersuchten und stets $0,07-0,11 \%$ gefunden haben, so ziemlich überein: von 0,08-0,13 auf 100. - Höhere Werthe habe ich nie gefunden. - Einzelne Bestimmungen des JugularisBlutes haben keinen wesentlichen Unterschied ergeben, wodurch die Resultate der von Pary und v. Mehring gemachten Zuckerbestimmungen eine neue Bestätigung erfahren.

Auffällig grosse Zahlen haben Böhm und Hoffmann (Arch.

1) Experimental-Studien über Diab. Berlin 1874. Verl. Oliven. p. 10. 
f. experiment. Pathol. Bd. VIII. Heft IV und V) für das Blut von Katzen gefunden. Ich glaubte diese Resultate dem Umstande zuschreiben zu müssen, dass die betreffenden Experimentatoren bei ihren Blutbestimmungen aus dem mit schwefelsaurem Natron gemischten Blute ungleich grössere Filtrate producirten, als es Bernard und Andre gethan haben. Ich überzeugte mich jedoch, dass ein dreimaliges Extrahiren mit kleinen Quantitäten kochenden Wassers ausreichte, um den Zucker dem Rückstande vollständig zu entziehen. Um ein Beispiel anzuführen, war für die Genauigkeit des Resultates bei einer Bestimmung von $15 \mathrm{grm}$. Blut ein Filtrat von $40-50$ grm. vollkommen genügend.

Noch über zwei andere Thatsachen musste ich mir Aufklärung verschaffen, bevor ich den Blutbestimmungen vollgültige Beweiskraft für die Resultate meiner Versuche einräumen durfte: 1) $0 \mathrm{~b}$ das Blut ron Kaninchen, deren Lebern glycogenfrei waren, Zucker enthalte? - Die Untersuchungen v. Mehring's ${ }^{1}$ ) haben in dieser Richtung ein positives Resultat ergeben, und stehen dieselben mit den Resultaten meiner Untersuchungen bei drei Kaninchen im Einklange, insofern dies einem Hungerzustande von 7 Tagen gilt; am 8., 9., und 10. Hungertage (die Thiere lebten meist 11-12 Tage) konnte ich keinen Zucker mehr nachweisen.

2) Ob die von Cl. Bernard mitgetheilten Erfahrungen, dass der Zucker des aus der Ader abgelassenen Blutes in relativ kurzer Zeit zerstört werde, zutreffen?

Vergleichende Untersuchangen zweier Blutproben haben mich belehrt, dass das Blut, in dem einen Falle 20, in dem andern 26 Minuten nach dem Aderlass, keinen nennenswerthen Untersehied in der Zuckermenge gegeniuber dem frischen Blute gezeigt hat.

Anknüpfend an die erste Versuchsreihe, deren Ergebnisse ich oben mitgetheilt habe, will ich mir nun erlauben, über die Versuche und die Methode

II. des pathologischen Experiments, (an vorher operirten Thieren)

die nöthigen Mittheilungen zu machen.

1) I. c. p. 415 . 
Wie ich bereits oben erwähnte, lag es im Plane dieser Arbeit, das Rückenmark in verschiedenen Höhen zu durchschneiden, und dann zu prüfen, wie sich diese schweren Eingriffe in den Organismus auf die Glycogenbildung der Leber äussern; wie sich jedoch aus der ersten Versuchsreihe ergiebt, hatte ich schon da den Kreis der Untersuchungen insofern erweitert, als ich nicht nur der Glyeogenbildenden Funktion in der Leber, sondern auch dem Schicksale des nicht za Glycogen umgewandelten Theiles der in den Körperkreislauf gelangten Zuckerlösung nachzuforschen mich bemühte. Dieser Vorgang wurde auch hier befolgt. Wegen der grossen Opfer, welche diese Experimente an Zeit, Mühe und Thieren erforderten, war ich ausser Stande, dieselben weiter als auf drei Versuchsreihen auszudehnen; diese beziehen sich

1. auf die Durchschneidung des Rückenmarks zwischen fünftem und sechstem Halswirbel;

2. auf die Durchschneidung zwischen letztem Hals- und erstem Brustwirbel und

3. auf die Durchschneidung zwischen zweitem und drittem Brustwirbel.

Jede Versuchsreihe umfasst acht Versuche. Was die Metho de betrifft, so habe ich ron dem Aufbinden des Kaninchens abgesehen und mich nach dem Vorgange von 0 . Israel ${ }^{1}$ ) sowohl bei der Durchschneidung zwischen fünftem und sechstem Halswirbel, als auch bei der zwischen letztem Hals- und erstem Brustwirbel eines einfachen Verfahrens bedient, indem ich direkt durch die Haut, ohne dieselbe erst zu trennen, durch den entsprechenden.Intervertebralraum ein schmales bauchiges Messerchen führte und damit das Mark trennte. Die Operation ist sehr leicht auszufuihren, und bedarf es nur einer Assistenz von 1-2 Minuten. Bei Durchschneidung zwischen zweitem und drittem Brustwirbel hielt ich es für nöthig, das Thier in der Bauchlage aufzubinden, um mit möglichster Schonung der Weichtheile die entsprechenden Dornfortsätze bloszulegen und in schräger Richtung entsprechend der Axe des Dornfortsatzes das Rückenmark zu durchschneiden. Nach vollzogener Operation wurde das Thier zum Zwecke der Temperatur-

1) Ueber künstliche Pökilothermie. Gekrönte Preisschrift. Arch. f. Anatomie und Physiologie. 1877. p. 443. 
Regulirung in den Wärmkasten gebracht, und daselbst nach 2-3 Stunden die Zuckereinspritzung in den bekannten Dosen gemacht. Nach 3-4 Stunden Abpressen des Harns und Tödtung durch Halsschnitt.

Bemerken muss ich jedoch, dass ich bei den meisten der folgenden Versuche gemischtes Blut und nur in seltenen Ausnahmen das der Carotis untersucht habe.

Bevor ich die nöthige Uebung hatte, kamen Täuschungen iiber den Effect der Operationen vor. Ich habe nämlich 2 bis 3 Stunden dauernde Lähmungen der, unterhalb der Durchschneidungsstelle innervirten Körpertheile gesehen, die, wie ich mich dann später zu überzeugen Gelegenheit hatte, eingetreten waren, ohne Continuitätstrennung des Riickenmarks und lediglich auf Compression desselben durch das im Wirbelkanal angehäufte Blut zurückzuführen waren; diese Versuche verliefen denn anch resultatlos. Selbstverständlich wurde nur auf solche Versuche Rücksicht genommen, bei welchen die postmortale Obduction ein positives Resultat für die vollständige Trennung des Marks geliefert hat.

Dass die verschiedenen Grade der ersten Reactions-Erscheinungen, wie: erhöhte Reflexthätigkeit der gelähmten Extremitäten, gesteigerte Athem- und Pulsfrequenz auf individuelle Eigenthümlichkeiten des Versuchsthieres zurückzufuthren seien, halte ich für wahrscheinlich, denn ich beobachtete dieselben nach vollkommen gelungenen Durchschneidungen, bei denen ich nach dem Aufhören der genannten Erscheinungen gleichmässigen Temperaturabfall (35-36) und verlangsamte Respiration (40-50) constatiren konnte.

Erwähnen möchte ich noch, dass ich in einzelnen Fällen beobachtet habe, dass Kaninchen mit durchschnittenem Mark im Strahle harnen und durch peristaltische Bewegungen des Mastdarms das in letzterem befindliche Thermometer ausstossen, Erscheinungen wie sie Goltz in seinem Vortrage (über die Functionen des Lendenmarks des Hundes, d. Arch. VIII. Pag. 474-482) erwähnt hat.

Nach diesen vorangeschickten Bemerkungen mögen nun in Tabelle II die Ergebnisse der Rückenmarks-Durchschneidung zwischen dem fünften und sechsten Halswirbel folgen. 
Beitrag zur Lehre von der Glycogenbildung in der Leber.

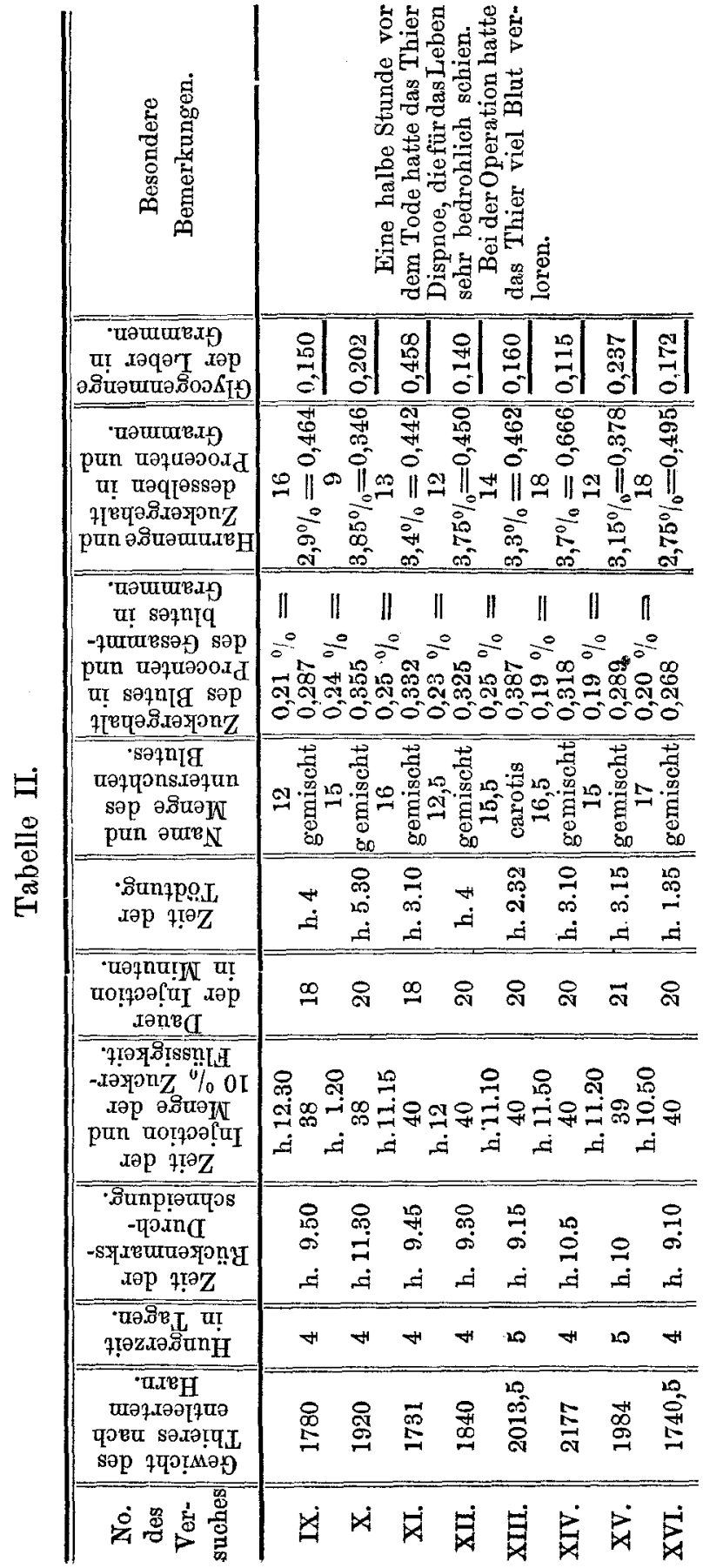


Vergleichen wir nun diese Tabelle mit der ersten, so fallen uns sofort die iiberraschend niedrigen Zahlen für die Glycogenmengen auf. Während für die erste Versuchsreihe sich ein Durchsehnitt von $0,723 \mathrm{grm}$. ergiebt, zeigt die II. Tabelle einen solehen von 0,202 grm.

In Nr. XI der II. Tabelle erseheint allerdings der hohe Glycogengehalt von 0,458 ; doch zusammengehalten mit dem Versuch Nr. II der I. Tabelle, bei dem sich die zweitgrösste Ziffer der ganzen Versuchsreihe zeigt, könnte man vielleicht die vorübergehende Dispnoe als Ursache heranziehen.

Nun miisste man glauben, dass diese grossen Differenzen in den Glycogenmengen durch entsprechend grosse Harnzuckerausscheidungen ausgeglichen werden sollten; das Gegentheil ist jedoch der Fall: die Durchschnittsmenge des Harnzuckers für die erste Versuchsreihe zeigt 1,33, für die zweite nur 0,463 .

Was die Zuckermenge des Blutes anbelangt, zeigen die Procentverhältnisse dieser beiden 'Versuchsreihen so geringe Unterschiede, dass sie sich vollständig decken.

Sehen wir nun in der folgenden Tabelle III, welche Ergebnisse die Durchschneidung zwischen letztem Hals- and erstem Brustwirbel liefert.

In dieser Versuchsreihe springen im Gegensatze zu den vorhergegangenen die hohen Glyeogenwerthe in die Augen, im Durchschnitt lieferte jeder Versuch 0,861 grm. Glycogen, eine Quantität, die sogar die Durchschnittsziffer der bei dem physiologischen Experiment (bei vorher nicht operirten Thieren) (Tab. I) erhaltenen Werthe uberragt. Es stehen diese Resultate mit denen von $\mathrm{Cl}$. Bernard bei Durchscheidung des Marks zwischen letztem Halsund erstem Brustwirbel gefundenen im Einklange.

Die ausgeschiedene Harnzuckermenge ist im Durchschnitt 0,409 und steht daher in einem ähnlichen Missverhältnisse zu der in der ersten Versuchsreihe gefundenen Durchschnittsziffer wie diejenige der II. Versuchsreihe.

Die Columne für die Znckerbestimmungen zeigt auch auf dieser Tabelle keine nennenswerthen Differenzen gegenüber denen der zwei ersten Tabellen.

Es erübrigt uns nun noch die Ergebnisse der IV. Versuchs- 
Beitrag zur Lehre von der Glycogenbildung in der Leber.

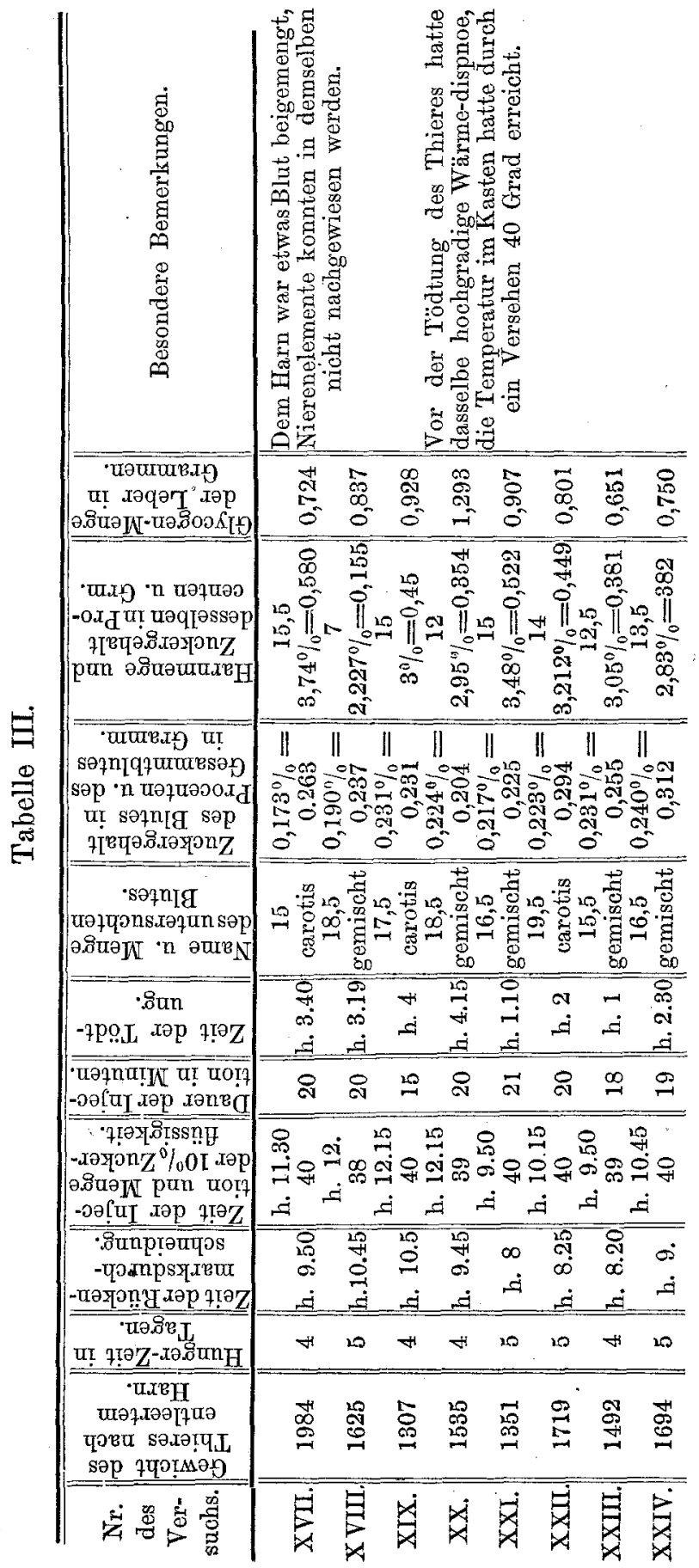




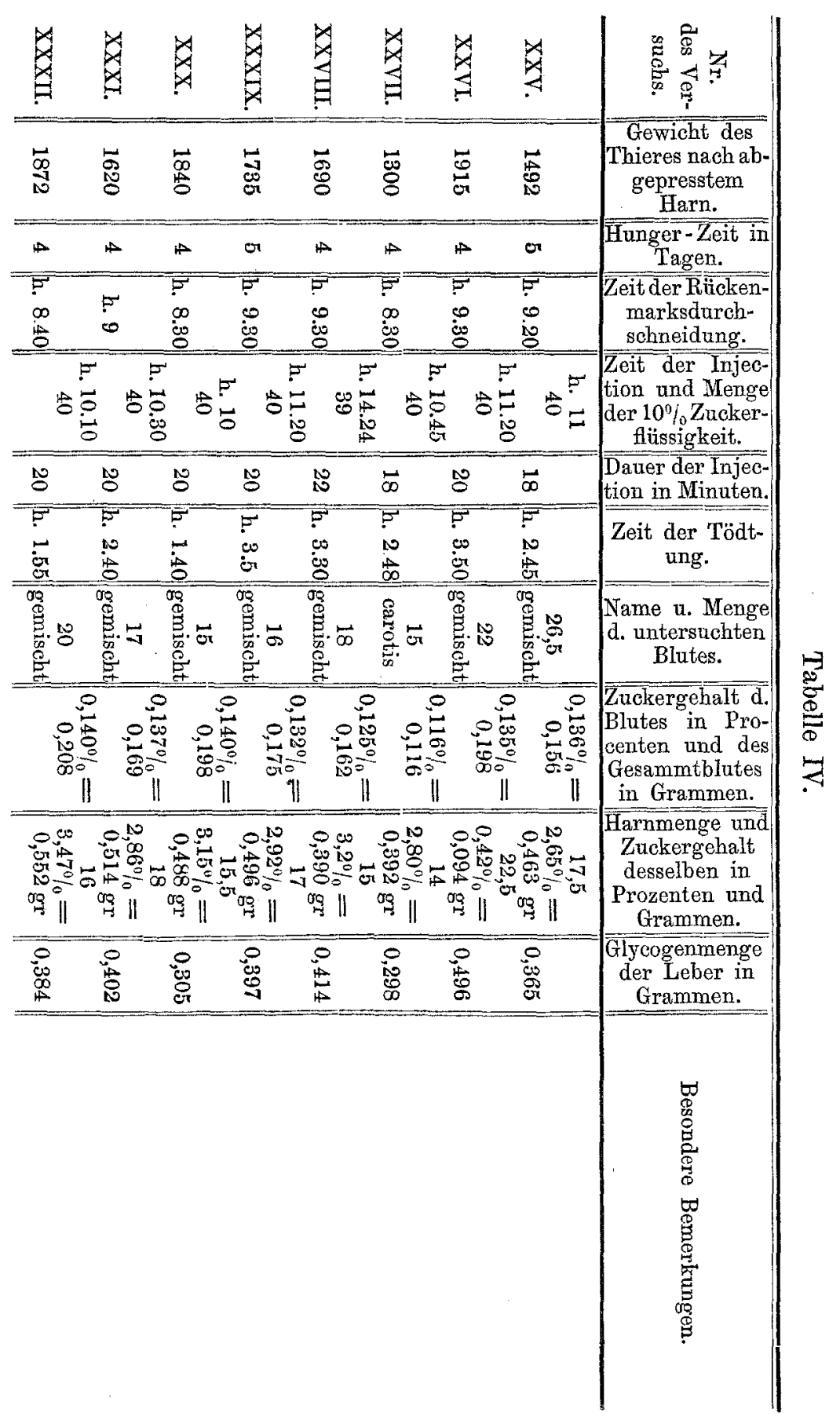


reihe mitzutheilen, die in der nebenstehenden Tabelle IV ihren Platz finden sollen.

Die Durchschnittsmenge für das Glycogen beträgt in dieser Versuchsreihe 0,383 grm. Die der ausgeschiedenen Harnzuckermenge $0,424 \mathrm{grm}$. und was den Zuckergehalt des Blutes anbelangt, so finden wir bei diesen Versuchen eine unzweifelhafte Abweichung von den in allen vorhergegangenen Fällen bestimmten Blutzuckermengen.

Wenn wir annehmen, dass bei vier- bis fünftägigem Hungern kräftige Kaninchen den Zuckergehalt ihres Blutes nur zum geringsten Theil verlieren, wie dies sowohl von v. Mehring, als auch von mir nachgewiesen wurde, so können wir aus den Resultaten dieser Versuchreihe ungezwungen den Schluss ziehen, dass die Thiere mit durchschnittenem Mark zwischen zweitem und drittem Brustwirbel von dem in ihren Körperkreislauf gebrachten Zucker zur Zeit ihrer Tödtung in dem Blute kaum noch etwas zurückbehalten haben, während sich in allen andern Versuchsreihen annähernd die doppelte Menge des von mir als normal gefundenen Zuckergehaltes vorgefunden hat.

Indem ich mich anschicke, zum Schlusse dieser Arbeit die Ergebnisse derselben zusammen zu fassen, bin ich mir wohl bewusst, dass bei der Komplicirtheit der von mir angestellten Versuche die grösste Vorsicht für die Schlussfolgerungen geboten ist.

Körpergewicht und Hungerzeit der Thiere der betreffenden Versuchsreihen war nicht vollständig gleich. Die Zeit der Injectionen schwankte um einige Minuten, die Tödtung erfolgte wenn auch annähernd, doch nicht zu ganz gleichen Zeitabläufen nach den vorhergegangenen Eingriffen. Dazu kommt der Uebelstand, dass es geradezu unmöglich ist, Fehlerquellen, die in den Untersuchungsmethoden gelegen sind, zu vermeiden.

Andererseits spricht die Gleichmässigkeit der gewonnenen Resultate je einer Versuchsreihe dafür, dass dieselben mit an Sicherheit grenzender Wahrscheinlichkeit auf den entsprechenden Eingriff zuriickzufiuhren sind.

Ich behalte mir vor diese Untersuchungen im Eingangs erwähnten Sinne zu geeigneter Zeit fortzusetzen. 
182 Jaques Mayer: Beitrag zur Lehre von der Glycogenbildung etc.

Ohne in den Folgerungen zu weit zu gehen, glaube ich aus den bis nun gewonnenen Resultaten folgende Schlïsse ziehen zu dürfen:

I. Rückenmarksdurchtrennung gleichviel ob

a. zwischen fünftem und sechstem Halswirbel,

b. zwischen letztem Hals- und erstem Brustwirbel,

c. zwischen zweitem und drittem Brustwirbel, verhindert nicht, dass der in den Kreislauf des Thierkörpers gebrachte Traubenzucker zum Theile in demselben zurückgehalten und im Stoffweehsel der Gewebe zur Verwendung komme.

II. Rückenmarks durchtrennung zwischen fünftem und sechstem Hals wirbel wirkt in beträchtlichem Grade hemmend auf die Glyeogenbildung (in der Leber) aus dem in den Körperkreislauf gelangten Zucker, ohnejedoeh vermehrte Zuckeransscheidung durch den Harn zu verursachen.

III. Durchtrennung des Marks zwisehen letztem Hals-und erstem Brustwirbel hat vermehrte Glycogenbildung (in der Leber) aus dem in den Körperkreislauf gebrachten Blute zur Folge, ohne dass der Zuckergehalt des Blutes vermindert wïrde.

IV. Durchtrennung des Rikkenmarks zwischen zweitem und drittem Brustwirbel hat verminderte Glycogenbildung (in der Leber) aus dem in den Körperkreislauf gebrachten Zucker zur Folge und verursacht eine beträchtliche Verwerthung des letzteren in den Geweben des Organismus.

Es ist mir eine angenehme Pflicht, Herrn Geheimrath, Professor Leyden für die Liberalität, mit der er mir die Benutzung seines Laboratoriums zu gestatten die Güte hatte, und für das lebhafte Interesse, mit dem er diese Arbeit begleitete und förderte, an dieser Stelle meinen wärmsten Dank auszusprechen. 